Correspondence

Nemat O. Keyhani

keyhani@ufl.edu

Received 13 January 2006

Revised 10 May 2006

Accepted 11 May 2006

\section{EST analysis of cDNA libraries from the entomopathogenic fungus Beauveria (Cordyceps) bassiana. I. Evidence for stage-specific gene expression in aerial conidia, in vitro blastospores and submerged conidia}

\author{
Eun-Min Cho, ${ }^{1}$ Li Liu, ${ }^{2}$ William Farmerie ${ }^{2}$ and Nemat O. Keyhani ${ }^{1}$ \\ Department of Microbiology and Cell Science ${ }^{1}$ and Interdisciplinary Center for Biotechnology \\ Research², University of Florida, Gainesville, FL 32611, USA
}

The entomopathogenic fungus Beauveria (Cordyceps) bassiana holds much promise as a pest biological control agent. $B$. bassiana produces at least three in vitro single cell infectious propagules, including aerial conidia, vegetative cells termed blastospores and submerged conidia, that display different morphological, biochemical and virulence properties. Populations of aerial conidia, blastospores and submerged conidia were produced on agar plates, rich liquid broth cultures and under conditions of nutrient limitation in submerged cultures, respectively. cDNA libraries were generated from mRNA isolated from each $B$. bassiana cell type and $\sim 25005^{\prime}$ end sequences were determined from each library. Sequences derived from aerial conidia clustered into 284 contigs and 963 singlets, with those derived from blastospores and submerged conidia forming 327 contigs with 788 singlets, and 303 contigs and 1079 contigs, respectively. Almost half $(40-45 \%)$ of the sequences in each library displayed either no significant similarity (e value $>10^{-4}$ ) or similarity to hypothetical proteins found in the NCBI database. The expressed sequence tag dataset also included sequences representing a significant portion of proteins in cellular metabolism, information storage and processing, transport and cell processes, including cell division and posttranslational modifications. Transcripts encoding a diverse array of pathogenicityrelated genes, including proteases, lipases, esterases, phosphatases and enzymes producing toxic secondary metabolites, were also identified. Comparative analysis between the libraries identified 2416 unique sequences, of which $20-30 \%$ were unique to each library, and only $\sim 6 \%$ of the sequences were shared between all three libraries. The unique and divergent representation of the $B$. bassiana transcriptome in the cDNA libraries from each cell type suggests robust differential gene expression profiles in response to environmental conditions.

\section{INTRODUCTION}

Insect fungal pathogens are dispersed within the major fungal taxonomic groups, and represent unique lifestyle adaptations that have evolved numerous times (Goettel et al., 2000; Khachatourians, 1996). Beauveria bassiana belongs to the order Hypocrealean within the Ascomycota, and is one of the most widely studied members of the Cordycipitaceae clade. B. bassiana has been environmental protection agency approved for commercial use against a

Abbreviations: EST, expressed sequence tag; GO, gene ontology.

The GenBank/EMBL/DDBJ accession numbers for the EST sequences reported in this paper are shown in the Supplementary Tables.

A figure and tables with further details about the EST data are available as supplementary data with the online version of this paper. variety of agricultural pests, including whiteflies, beetles, grasshoppers and psyllids. B. bassiana has also been used as a model system to study fungal-mediated tick (Acari: Ixodidae) biological control, and recent studies have highlighted the potential for entomopathogenic fungi, including $B$. bassiana, as agents in combating the spread of malaria by controlling mosquito populations (Blanford et al., 2005; Kirkland et al., 2004; Scholte et al., 2005). The Beauveria lifestyle is further unique in that it is a facultative saprophyte and can exist as a plant endophyte and/or form intimate interactions with plant roots (Bing \& Lewis, 1992; Elliot et al., 2000; Lewis et al., 2001; Wagner \& Lewis, 2000; White et al., 2002).

B. bassiana has evolved sophisticated mechanisms for penetrating the formidable barrier that constitutes the 
insect/arthropod exoskeleton or cuticle. (Binnington \& Retnakaran, 1991; Boman, 1981; Clarkson \& Charnley, 1996; Ferron, 1981; Neville, 1975; St Leger, 1991). Interspersed within the cuticle barrier are biochemical components, such as toxic lipids and phenols, enzyme inhibitors, proteins and other defensive compounds that entomopathogens must overcome for successful virulence (Anderson et al., 1995; Hackman, 1984; Renobales et al., 1991). Pathogens must cope with hydrophobic barriers, electrostatic charges, low relative humidity, low or sequestered nutrient levels, endogenous microbial flora, and crosslinked proteins that contribute to a stiff and desiccated cuticle (St Leger, 1991). Successful pathogenic fungi must also thwart infection-induced responses such as melanization and haemocyte activation (Boman, 1981; Pendland \& Boucias, 1985, 1986; Pendland et al., 1993; Riley, 1997).

The overall process of arthropod infection by pathogenic fungi involves many steps (Boucias \& Pendland, 1991; Carruthers \& Soper, 1987; Charnley, 1990; Charnley \& St Leger, 1991) most of which remain unelucidated, particularly at the molecular level. Entomopathogenic fungi possess complex systems for (1) finding the appropriate insect host(s), (2) adhering to the exoskeletal substrata, (3) evading host defences, (4) penetrating and degrading the cuticle, (5) transporting to the cytoplasm and catabolizing necessary nutrients (carbon/nitrogen, external products of the degradation), and (6) dispersing from the catabolized host(s). The proteins and enzymes that contribute to this process include those that are secreted, and those that are found in the cell envelope, vesicles and vacuoles, as well as the cytoplasm and nucleus. B. bassiana does not require an opening or ingestion for attachment or germination, and is presumably able to penetrate anywhere on the cuticle surface (although preferential sites of infection have been described for some insects).

$B$. bassiana produces at least three single cell infectious propagules, known as aerial conidia, in vitro blastospores and submerged conidia, which display different morphological and biochemical properties (Bidochka et al., 1987; Jeffs et al., 1999; Thomas et al., 1987). Aerial conidia are relatively resistant to varying environmental conditions and represent the most commonly used cell form in biological control applications. Aerial conidia contain a rodlet layer that presumably contributes to the hydrophobic nature of these cells (Holder \& Keyhani, 2005). In contrast, blastospores are hydrophilic, do not appear to contain a rodlet layer and bind poorly to hydrophobic surfaces, but germinate and grow at much higher rates than aerial conidia. Submerged conidia are also hydrophilic, but appear to be able to bind to both hydrophobic and hydrophilic surfaces, contain a rough surface morphology, and may be an important developmental adaptation for growth under nutrient limiting conditions.

B. bassiana contains a haploid nuclear genome of between 34 and $44 \mathrm{Mb}$, consisting of 7-8 chromosomes, which by comparison to equivalent fungal genomes would contain
10 000-15 000 genes (Pfeifer \& Khachatourians, 1993; Viaud et al., 1996). We have employed an expressed sequence tag (EST) analysis of aerial conidia, in vitro blastospores and submerged conidia in order to characterize the B. bassiana transcriptome. Analysis of the libraries revealed a robust diversity in expressed transcripts, with the majority of sequenced ESTs unique to each library. These data will provide a basis for investigating the gene expression profile of distinct developmental stages as well as the overall genomic potential of $B$. bassiana. It also will facilitate gene identification, ORF calling and mRNA processing site identification for B. bassiana genome sequencing efforts.

\section{METHODS}

Cultivation of fungi. B. bassiana (ATCC 90517) was routinely grown on potato dextrose agar (PDA). Plates were incubated at $26^{\circ} \mathrm{C}$ for $10-14$ days and aerial conidia were harvested by flooding the plate with sterile $\mathrm{dH}_{2} \mathrm{O}$. Conidial suspensions were filtered through a single layer of Miracloth and final spore concentrations were determined by direct count using a haemocytometer. Blastospores were produced in Sabouraud dextrose/0.5-1\% yeast extract (SDY) liquid broth cultures inoculated with conidia $\left(0 \cdot 5-5 \times 10^{5}\right.$ conidia $\mathrm{ml}^{-1}$ final concentration). Cultures were grown for 3-4 days at $26^{\circ} \mathrm{C}$ with aeration (shaking incubator, 150 r.p.m.). Cultures were filtered (twice) through glass wool to remove mycelia, and the concentration of blastospores determined by direct count. Submerged conidia were produced in TKI broth using fructose as the carbon source (Thomas et al., 1987). For all cell types, Miracloth or glass wool filtered cell suspensions were harvested by centrifugation $\left(10000 \mathrm{~g}, 15 \mathrm{~min}, 4^{\circ} \mathrm{C}\right)$, washed twice with sterile $\mathrm{dH}_{2} \mathrm{O}$ and resuspended at $10^{7}-10^{8}$ cells $\mathrm{ml}^{-1}$.

Construction of cDNA libraries. Fungal cells were lysed by grinding in liquid nitrogen and total RNA was extracted using RNAWiz (Ambion). Partially purified mRNA was recovered from the total RNA pool using the Poly(A)Purist mRNA purification system (Ambion), both according to the manufacturer's recommendations. cDNA libraries were constructed using the unidirectional pBluescript II XR system (Stratagene). Colonies picked for sequencing ( $\sim 2500$ per library) were derived from primary libraries containing $0 \cdot 5-1 \cdot 0 \times 10^{6}$ c.f.u. without amplification.

Plasmid isolation and DNA sequencing. Plasmid constructs were transformed into XL10-Gold ultracompetent cells (Stratagene) and the presence of inserts selected for using the blue/white screening on $\mathrm{X}-\mathrm{Gal} / \mathrm{LB}$ agar plates. Randomly selected bacterial cDNA clones were grown in 96-well microplates overnight in LB/8\% glycerol. A small portion of each bacterial culture was used for sequence template production and the remainder of the culture retained in a frozen archive. All sequencing templates were prepared using Amersham Biosciences TempliPhi rolling circle DNA amplification kits as specified by the supplier, with minor protocol modification. For DNA sequencing reactions Amersham Biosciences ET Terminator dye terminator cycle-sequencing chemistry was used. The reactions were precipitated with ethanol to remove unincorporated terminator, resuspended in loading solution and analysed by capillary gel electrophoresis using Amersham MegaBACE 1000 DNA sequencing units. All DNA sequence electropherogram files were processed by a Geospiza Finch Suite DNA sequencing management system (version 2.10.1) for base calling by Phred (version 0.020425.c) and archival storage of sequence electropherograms.

Sequence analysis. EST-sequence clustering and assembly was performed using TranscriptAssembler (PTA) version 2.7.0 (Paracel). 
The SCYLLA component of PTA masked vector sequences, filtered Escherichia coli chromosomal DNA contamination, filtered rRNA components, trimmed low quality bases from the ends of EST sequences and annotated intrinsic repeats such as simple sequence repeats and SINE elements prior to clustering and assembly. EST sequences with fewer than 100 high quality nucleotides were excluded from the clustering and assembly steps. After filtering, EST sequences were passed to the PTA clustering module for pairwise comparison. EST sequences with a minimum Smith-Waterman score of 50 were placed together in individual clusters. Each cluster of related EST sequences was assembled using the CAP4-based PTA assembly module. All BLAST searches were conducted using a local compute cluster using Paracel BLAST (version 1.5.6). The results were parsed and stored in a local SQL database that facilitated search and summary of BLAST results on the basis of user-defined criteria through a web browser-based interface.

Gene ontology (GO) assignments. For functional classification according to GO assignments, all contig and singlet sequences were queried against the NCBI non-redundant (NR) protein database using BLASTX with the $e$ value threshold set to $\leqslant 10^{-4}$. The top 100 BLAST hits were selected for further analysis. GO terms associated with the best scoring BLAST hits were assigned back to the original query nucleotide sequence. Based on these GO term assignments, the complete UniGene EST set for the cDNA library was organized around GO hierarchies divided into biological processes, cellular components and molecular functions (Harris et al., 2004). BLAST results were parsed and stored in BlastQuest, a SQL database that facilitates BLAST results management and GO term browsing (Farmerie et al., 2005).

\section{RESULTS AND DISCUSSION}

\section{Characterization of the EST database}

EST libraries were constructed from mRNA derived from $B$. bassiana aerial conidia, in vitro blastospores and submerged conidia. Cell preparations were deemed greater than $95 \%$ homogeneous as determined by microscopic analysis of samples used for RNA extraction. Approximately 2500 clones were picked at random from each library and subjected to single pass $5^{\prime}$ sequencing. The mean insert size of the cDNA libraries ranged from 0.8 to $1.2 \mathrm{~kb}$ with an overall range of $0 \cdot 4-2 \cdot 8 \mathrm{~kb}$. After editing the mean length of the single read sequences ranged from 0.4 to $0.5 \mathrm{~kb}$. An analysis of the total number of runs, the number of valid ESTs, assembly of the ESTs into groups of cDNAs that share sequence identity and are considered to represent transcripts of the same gene (contigs), the number of singlets, and the diversity index for each library is given in Table 1. The representative nature of the libraries was confirmed by the high degree of transcript diversity (diversity index ranging from 57 to $66 \%$ ). The distribution of sequences per contig is presented in Fig. 1. No EST obviously representing rRNA or prokaryotic contamination was found in any of the sequences (prior to filtering).

\section{Functional analysis of the EST libraries}

The clustered and singlet sequences constituting the unigene set of each library were used for BLASTX searches. Significant hits were defined based upon $e$ value scores $\leqslant 10^{-4}$, which identified contigs with amino acid sequence similarity to previously characterized proteins deposited in the GenBank NR and/or Swissprot databases (as of September 2005). Search results were also used to extract gene annotation terms for grouping sequences into functional classifications. The complete search results, with GenBank accession numbers, for each EST are available as supplementary data with the online journal (Supplementary Table S1).

The number of contigs that displayed matches to proteins in the GenBank/Swissprot databases (September 2005) were 581 out of 1247 ESTs ( $47 \%), 508 / 1115(46 \%$ ) and 725/1382 $(52 \%)$, for the aerial conidia, blastospore and submerged conidia libraries, respectively. Of the ESTs with significant matches, $75-90 \%$ could then be assigned GO terms. Most (70-80\%) of the ESTs displayed a fungal sequence as the best match, with the greatest number of hits among ascomycete sequences and only $1-4 \%$ among the basidiomycetes. ESTs displaying similarity to sequences from animals or prokaryotes represented $8-10 \%$ of the total, and less that $5 \%$ showed similarity to plant, archaeal or protist sequences.

GO representations of the unigene EST set for each library were constructed around the GO organizing hierarchies of (i) biological processes (Figs 2a, 3a, 4a), (ii) cellular components (Figs $2 \mathrm{~b}, 3 \mathrm{~b}, 4 \mathrm{~b}$ ) and (iii) molecular functions (Figs 2c, 3c, 4c) (see Supplementary Tables S2 and S3 available with the online journal). These tables and figures

Table 1. Summary of $B$. bassiana EST sequencing data

\begin{tabular}{|lccc|}
\hline & Aerial conidia & Blastospore & Submerged conidia \\
\hline Total no. of runs & 2380 & 2329 & 2540 \\
Total no. of valid ESTs & 1952 & 1947 & 2092 \\
Total no. of contigs & 284 & 327 & 303 \\
No. of EST in contigs & 989 & 1159 & 1012 \\
Total no. of singlets & 963 & 788 & 1079 \\
Total no. of unique sequences & 1247 & 1115 & 1382 \\
Diversity index $(\%)$ & 64 & 57 & 66 \\
\hline
\end{tabular}

${ }^{\star}$ Diversity index $=$ total no. of unique sequences/total no. of valid ESTs. 

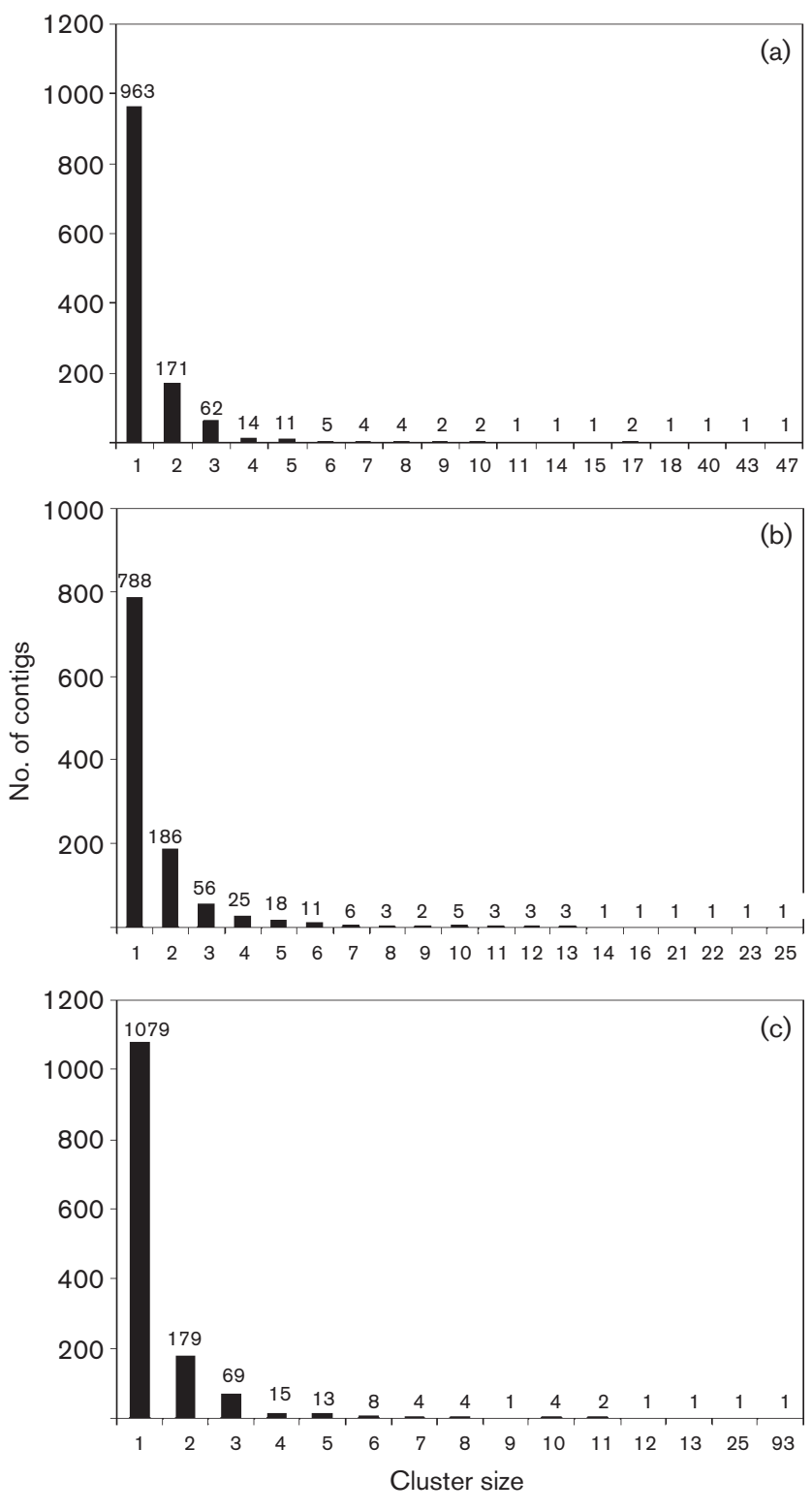

Fig. 1. EST redundancy in contig assembly: (a) aerial conidia, (b) blastospores and (c) submerged conidia.

provide a representation by major $\mathrm{GO}$ categories including (i) metabolism, nutrient uptake and cell communication; (ii) cytoplasmic, nuclear, membrane and extracellular localization; (iii) ligand binding; (iv) catalytic, transport and signal transduction activities.

\section{Comparative analysis between the aerial conidia, in vitro blastospore and submerged conidia libraries}

Functional categorization of the ESTs isolated from aerial conidia, in vitro blastospores and submerged conidia revealed similar representations of most metabolism categories including protein, phosphorus, nucleic acid, carbohydrate, and amino acid catabolism and biosynthesis. Likewise, the distribution of sequences within ribosomal, cytoskeletal, mitochondrial, nuclear and membrane intracellular subcategories, as well as between enzymic functional categories, were similar between the three libraries (see Supplementary Fig. S1 available with the online journal). Although this global analysis revealed similar distributions within these functional categories, few of these represented overlapping genes (i.e. instead we observed different genes that were categorized within the same functional group).

An overall analysis of unique and shared sequences between the libraries was performed (Fig. 5). In all, 2193 different transcripts were identified between the three libraries. The library generated from submerged conidia contained the largest number of unique sequences (739 corresponding to $30 \%$ of the total clustered unigene set), with unique aerial conidial sequences representing $26 \%$ of the total unique gene set and blastospores accounting for $21 \%$ of the total.

An analysis of the 10 most abundant ESTs found in each dataset is presented in Table 2 (for the top 25 most abundant transcripts see Supplementary Table S4 available with the online journal). For comparative purposes these tables also list the number of times each sequence was found in the other libraries. Overall, the top 25 most highly represented transcripts accounted for between 15-17\% of the total ESTs in the datasets for each library.

Aerial conidia. Aerial conidia are produced sympodially, in acropetal succession on elongating conidiogenous cells from hyphae and mycelia growing on solid surfaces (Hegedus et al., 1992; MacLeod, 1954). Due to their spore-like qualities and relative greater stability and resistance to environmental conditions, these cells are often used as the infectious agents for biological control. Thus, much effort has gone into creating stable formulations and optimizing large scale production and delivery protocols (Desgranges et al., 1993; Inglis et al., 1995; Lord, 2001; Wraight et al., 2001). A list of the clone IDs, BLAST scores and top search result of the ESTs described in the following paragraphs have been complied in Supplementary Table S5 available with the online journal. A hydrophobin was the most highly represented transcript in both the aerial conidia and submerged conidia datasets (and second in representation in the blastospore EST library). Hydrophobins are unique small molecular mass fungal proteins, and different members of this protein family play roles in a diverse range of physiological processes, including adhesion, development (formation of aerial structures) and pathogenesis (Linder et al., 2005; Wosten, 2001). The aerial conidia library contained a number of unique ESTs similar to depolymerizing enzymes, including (i) proteases and peptidases, e.g. subtilisin protease, tripeptidyl-peptidase and an EST similar to a thermophilic serine protease from Bacillus Ak.1 (MacIver et al., 1994), (ii) glycosidases, e.g. N,O-diacetylmuraminidase and chitinase, and (iii) lipases, and an EST similar to 
(a) Biological processes

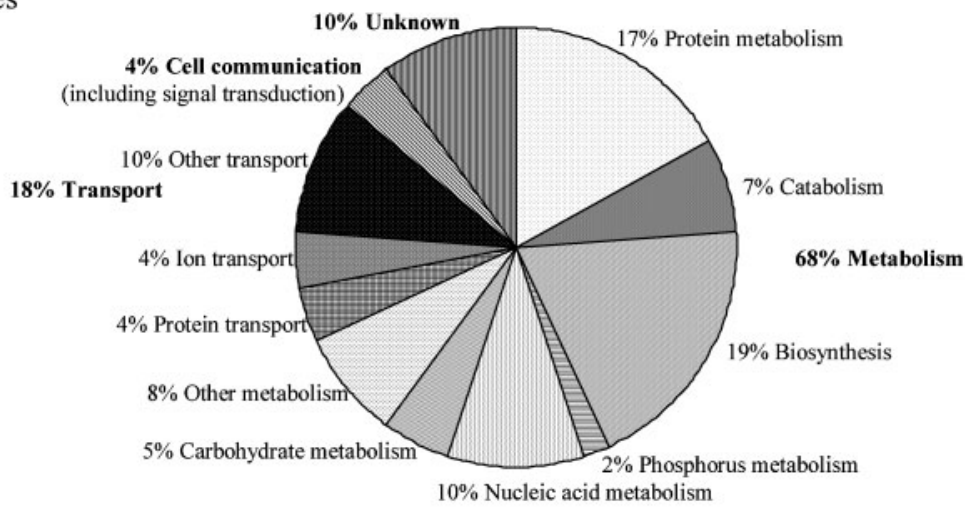

(b) Cellular components

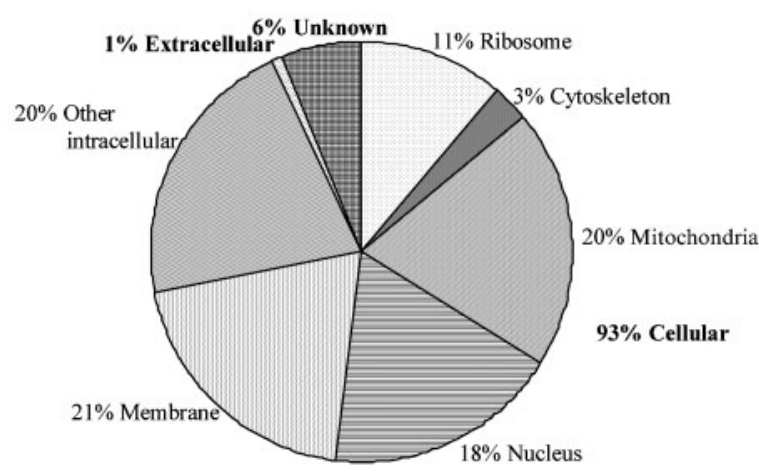

(c) Molecular functions

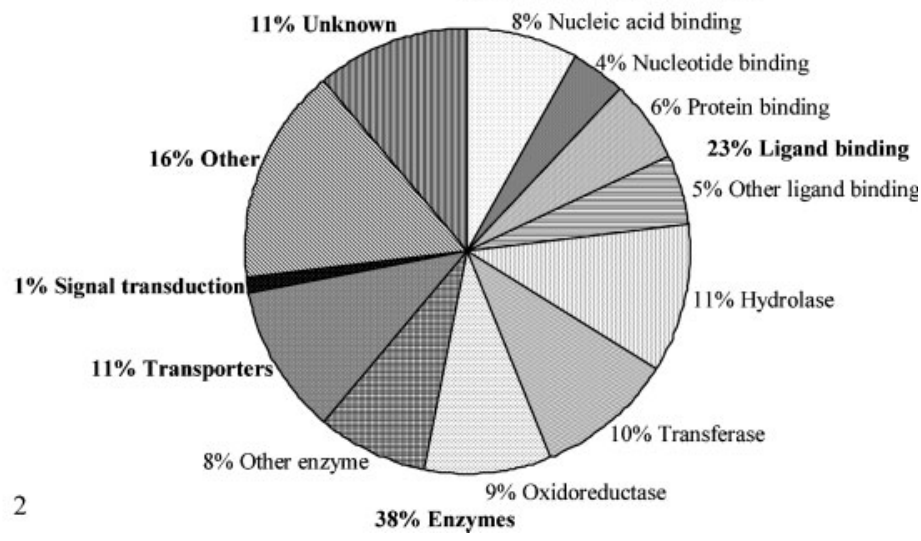

Fig. 2. Percentage representation of GO mappings for $B$. bassiana ESTs derived from the aerial conidia cDNA library.

the platelet-activating factor acetylhydrolase. This secreted phospholipase (A2) is unusual in that it is calcium dependent and contains amino acid motifs characteristic of neutral lipases and serine esterases (Tjoelker et al., 1995). These enzymes play important roles in fungal virulence towards arthropods (El-Sayed et al., 1993; Freimoser et al., 2003, Charnley \& St Leger, 1991; Gupta et al., 1994) and our data suggest that conidia (the dispersive spores) are pre-loaded with some of the enzymes (or with transcripts at least) that are required for cuticle degradation and establishment of the fungal infection on target hosts. The aerial conidia library contained transcripts potentially involved in spore cell wall formation including an EST similar to the sporulation specific 1,3 - $\beta$-glucan synthase from Schizosaccharomyces pombe (Martin et al., 2000) and a class V chitin synthase, as well as a septin homologue (Homann et al., 1996) possibly involved in cell surface organization. A diverse number of cytochrome p450 ESTs were also found, including a sterol $\alpha$-14-demethylase, an alkane hydroxylase, a trichodiene oxygenase, a sterol 26-hydroxylase, an enzyme similar to the plant (Arabidospsis thaliana) 89A2 p450 and a bifunctional p450/ NADPH-p450 reductase, indicating the availability of a robust detoxification and cell defence response. In terms of secondary metabolism, transcripts similar to the
Gibberella fujikuroi farnesylpyrophosphate synthase, a key enzyme in isoprenoid biosynthesis leading to the biosynthesis of several classes of essential (terpenoid) metabolites, including sterols, quinones, carotenoids and gibberellins (Homann et al., 1996), was identified, along with an enterobacterial aerobactin siderophore biosynthesis protein (Martinez et al., 1994). A number of ESTs related to drug resistance and remediation were identified. These included a transcriptional activator containing a $\mathrm{Zn}_{2} \mathrm{C}_{6}$-type zinc finger motif required for the induction of fluconazole resistance (Talibi \& Raymond, 1999), a mitomycin oxidase (August et al., 1994), a multidrug ABC transporter and a salicylate hydroxylase, which catalyses the formation of catechol from salicylate (You et al., 1991), a key intermediate in naphthalene catabolism.

In vitro blastospores. In vitro blastospores represent a single celled vegetative state produced in rich nutrient broth. Depending upon nutrient conditions, these cells are released laterally as well as terminally from growing hyphae (Bidochka et al., 1987). In vitro blastospores are cylindrical, somewhat irregular in size, smooth walled (i.e. no visible rodlet layer) infectious propagules that can germinate to form new hyphae or under certain nutrient conditions can produce microcycle conidia. Unlike aerial 
(a) Biological processes

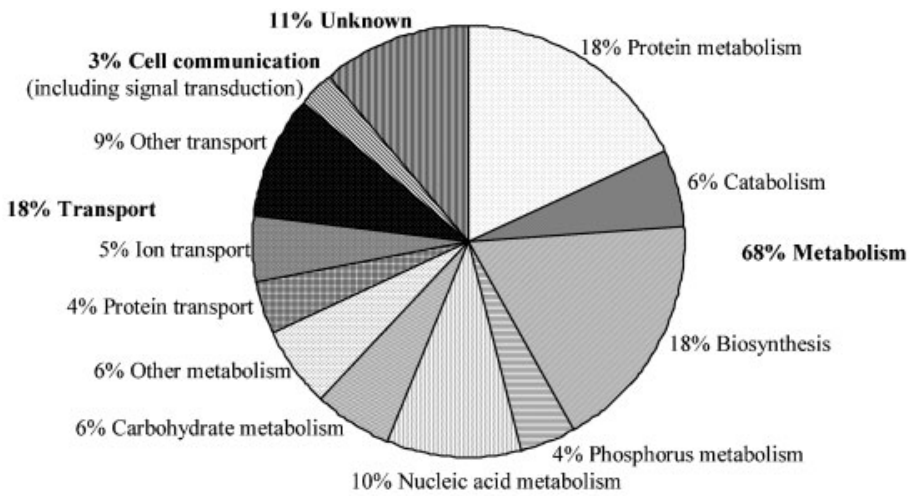

(b) Cellular components

(c) Molecular functions

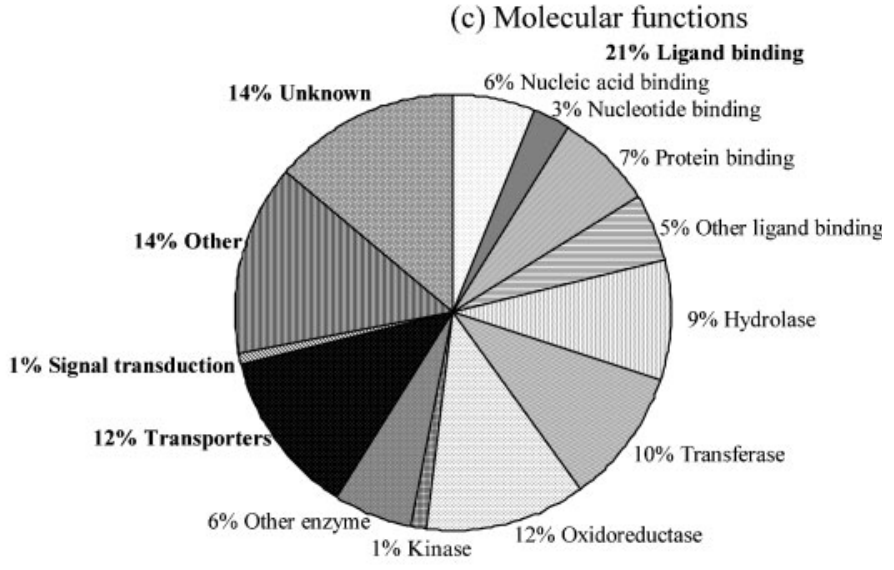

$38 \%$ Enzymes

Fig. 3. Percentage representation of GO mappings for $B$. bassiana ESTs derived from the blastospore cDNA library.

conidia, the blastospore EST collection contained few hydrolases, although a number of unique enzymes were noted. These included (i) peptidases, represented by two different aminopeptidases, one an EST similar to the secreted acid protease from the yeast Saccharamycopsis fibuligera (Hirata et al., 1988) and one an EST displaying similarity to the avian aminopeptidase H/bleomycin hydrolase (Adachi et al., 1997), (ii) cellulolytic glycosidases, one similar to the Trichoderma reesei cellulase and another EST similar to the Aspergillus aculeatus cellobiase, and (iii) an EST similar to the Penicillium notatum phospholipase enzyme that displays intrinsic lysophospholipase and phospholipase B activities (Masuda et al., 1991). Since blastospores are infectious agents, the lack of hydrolases may imply either a rapid induction of these enzymes when in contact with hosts or that some of these enzymes are present (translated) even though their transcripts were not found in our dataset. The blastospore library contained a number of ESTs encoding transporters. These included ESTs displaying similarity to a high affinity methionine permease, the Pseudomonas putida phthalate transporter, a mammalian chloride channel, a putative yeast copper transporter and the Aspergillus nidulans mirB triacetylfusarinine $C$ siderophore transporter. Vegetative incompatibility regulates the fusion of hyphae, which is generally followed by the exchange of cytoplasm and nuclei. ESTs displaying similarity to the Podospora anserina vegetative incompatibility protein HET-E-1 were identified (Saupe et al., 1995). Finally, an EST with similarity to a novel antifungal peptide presumed to be an important element of the innate immunity system from the insect Acrocinus longimanus (Coleoptera) was identified (Barbault et al., 2003).

Submerged conidia. The production of submerged conidia by $B$. bassiana represents an adaptation to nutrient-stress conditions. These cells are smaller and more uniform in size than blastospores, spherical in shape, refractile or hyaline, and have a rough surface morphology with no rodlet bundles (Bidochka et al., 1987; Holder \& Keyhani, 2005). Two different modes of submerged conidia production have been noted, (a) sporogenous cells occurring on mycelium or on short branches of hyphae and (b) germinating blastospores that give rise to (submerged) conidia at the tip of short germ tubes (Bidochka et al., 1987). These cells are infectious and germinate slightly faster than aerial conidia. With respect to development, cell wall remodelling and nutrient uptake, the submerged conidia library contained unique ESTs similar to (a) the catalase peroxidase (catalase 2) of 
(a) Biological processes

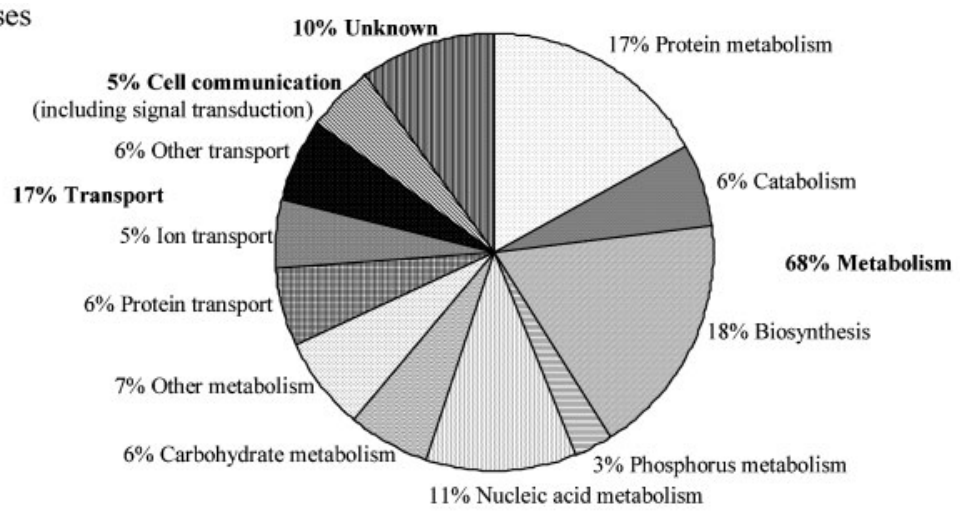

(b) Cellular components

(c) Molecular functions
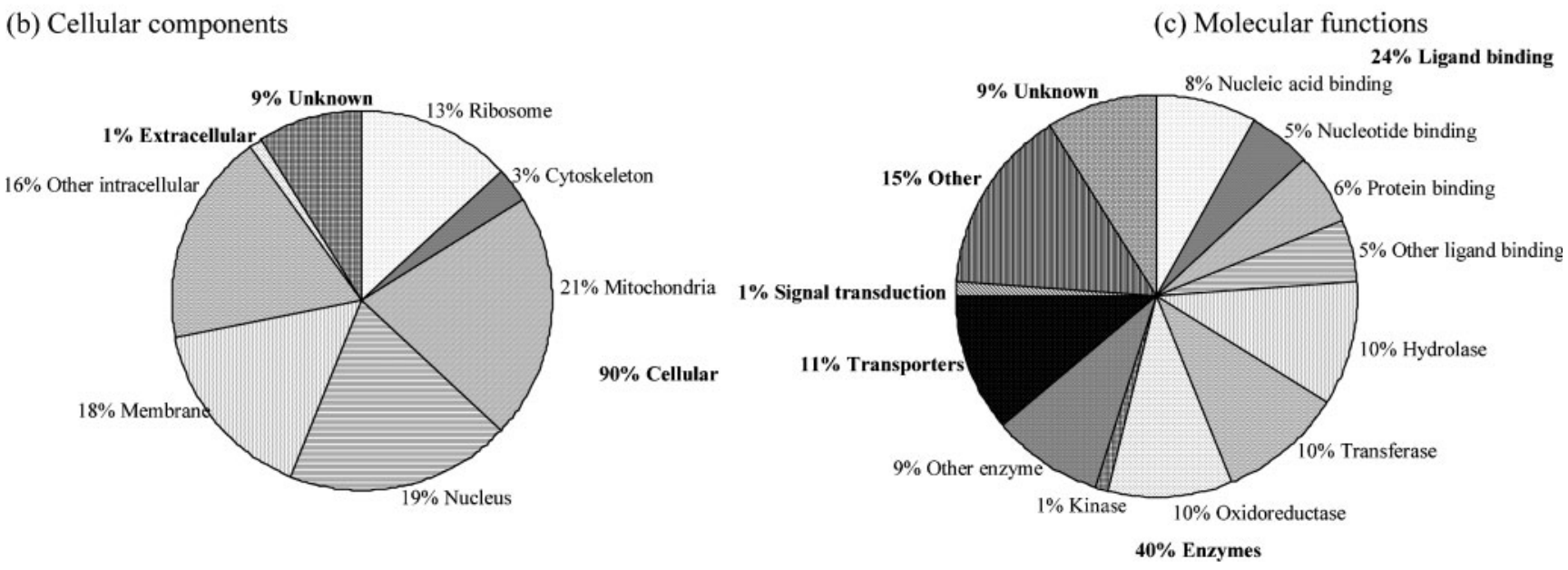

Fig. 4. Percentage representation of GO mappings for $B$. bassiana ESTs derived from the submerged conidia cDNA library.

Neurospora crassa implicated in cell differentiation and fungal morphogenic transitions (Peraza \& Hansberg, 2002), (b) the 1,3- $\beta$-glucan synthase component, Bgs4, of

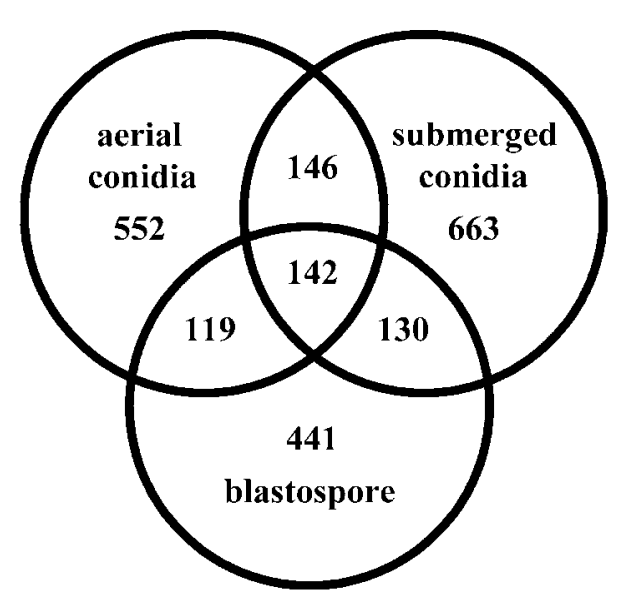

Fig. 5. Comparative analysis of sequenced ESTs derived from the $B$. bassiana aerial conidia, blastospore and submerged conidia cDNA libraries: the Venn diagram shows unique and overlapping sets of transcripts between the libraries.
Schizosaccharomyces pombe and the chitin synthase B from A. nidulans (Yanai et al., 1994), and (c) the Candida albicans peptide transporter, PTR2 (Basrai et al., 1995) and mammalian pepsinogen/gastricsin. Two ESTs within the submerged conidia library corresponded to parts of the 25 gene sterigmatocystin cluster of $A$. nidulans (Brown et al., 1996), including a moderately represented EST cluster (four sequences) similar to the A. nidulans verA gene product that converts the aflatoxin precursor versicolorin $A$ to sterigmatocystin, and a sterigmatocystin biosynthesis monooxygenase. Intriguingly, another EST was similar to the mammalian aflatoxin (B1) aldehyde reductase, a putative detoxifying enzyme (Knight et al., 1999), suggesting that $B$. bassiana possess mechanisms for both production and protection from toxins. Submerged conidia also expressed a number of efflux pump genes including ESTs similar to the Cochliobolus carbonum cyclic peptide efflux pump, ToxA, to a Schizosaccharomyces pombe multidrug efflux transporter similar to mammalian p-glycoproteins, implicated in mediating leptomycin B resistance, and to the Pur8 protein from Streptomyces alboniger that confers puromycin resistance (Tercero et al., 1993). Finally, the submerged conidia library contained an EST similar to mammalian soluble epoxide hydrolase, an enzyme involved 
Table 2. The most abundantly represented transcripts in the $B$. bassiana aerial conidia, in vitro blastospore and submerged conidia libraries

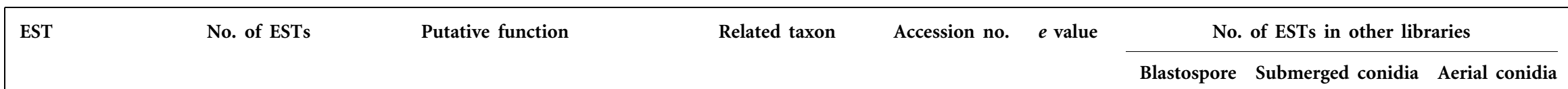

\section{Aerial conidia}

conidia.014.c1

conidia.009.c1

conidia.021.cl

conidia.059.c1

conidia.020.c1

conidia.078.c1

conidia.024.c1

conidia.028.c1

conidia.131.c1

conidia.034.c1

conidia.062.cl

In vitro blastospore

blastospores.030.cl

blastospores.081.c2

blastospores.005.cl

blastospores.099.c3

blastospores.048.c1

blastospores.024.c1

blastospores.014.c1

blastospores.028.cl

blastospores.050.cl

blastospores.003.cl

blastospores.116.c1

blastospores.123.c1

Submerged conidia

TKI.001.c1

TKI.024.c1

TKI.039.c1

TKI.017.c1

TKI.048.c1

TKI.080.c1

TKI.015.c1

TKI.053.c1

TKI.098.c1

$\begin{array}{lc}47 & \text { Rodlet protein precursor (rodA) } \\ 43 & - \\ 40 & - \\ 18 & - \\ 17 & - \\ 17 & \text { Elongation factor } 1-\alpha \text { (TEF) } \\ 15 & \text { ADP/ATP carrier protein (acp) } \\ 14 & - \\ 11 & \text { Hypothetical UPF0057 protein } \\ & \text { T23F2.5 in chromosome X }\end{array}$

10

10

T23F2.5 in chromosome $\mathrm{X}$$$
-
$$

Rodlet protein precursor (rodA)

Glucose-repressible gene protein (grg-1) Neurospora crassa

$$
-
$$

Hypothetical $34 \cdot 1 \mathrm{kDa}$ protein

in WHI3-CHS1 intergenic region

Clock-controlled protein 6 (ccg-6)

Histone H3 (hh3)

ADP/ATP carrier protein (acp)

$$
-
$$

Nucleoside diphosphate kinase $(\mathrm{swoH})$

Elongation factor $1-\alpha$ (TEF)

Rodlet protein precursor (rodA)

Elongation factor $1-\alpha$ (TEF)

Clock-controlled protein 6 (ccg-6)

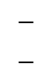

$-$

Aquaporin-like protein (AQY1)

Alcohol dehydrogenase I (adh-1)
Aspergillus nidulans

$$
-
$$

Podospora anserina

Neurospora crassa

Caenorhabditis elegans

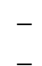

Aspergillus nidulans

Saccharomyces cerevisiae

Neurospora crassa

Neurospora crassa

Neurospora crassa

$$
-
$$

Aspergillus nidulans

Podospora anserina

Aspergillus nidulans

Podospora anserina

Neurospora crassa

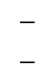

$-$

Saccharomyces cerevisiae Neurospora crassa

$\begin{array}{ccr}\text { P28346 } & 1 \cdot 91 e^{-11} & 23 \\ - & - & 0 \\ - & - & 0 \\ - & - & 0 \\ - & - & 0 \\ \text { Q01520 } & 0 & 12 \\ \text { P02723 } & 2 \cdot 16 e-42 & 13 \\ - & - & 0 \\ \text { Q22702 } & 1 \cdot 94 e^{-14} & 10\end{array}$

93

1

6

0

25

0

12

$\begin{array}{lll}- & - & 0\end{array}$

$\begin{array}{cc}- & - \\ \text { P28346 } & 1 \cdot 85 e-11 \\ \text { P22151 } & 1 \cdot 38 e^{-18} \\ - & - \\ \text { P40169 } & 2 \cdot 86 e-13 \\ & \\ \text { Q01302 } & 6 \cdot 55 e-04 \\ \text { P07041 } & 0 \\ \text { P02723 } & 0 \\ - & - \\ - & - \\ \text { Q8TFN0 } & 0 \\ \text { Q01520 } & 0\end{array}$

-, No significant search results for query $\left(e\right.$ value $\left.>10^{-3}\right)$. 
in detoxification of environmental mutagens and carcinogens playing an important role in xenobiotic metabolism by degrading potentially toxic epoxides.

Shared transcripts. The ESTs shared between only the aerial conidia and blastospores accounted for $4.9 \%$ (119/ 2416) of the total unigene set. This common set included a number of ABC-type multidrug transporters, an EST similar to the Schizosaccharomyces pombe multidrug resistance transporter conferring brefeldin A resistance (Nagao et al., 1995), and an EST similar to the Saccharomyces cerevisiae PDR5 protein that confers resistance to cycloheximide, sporidesmin and other mycotoxins.

Blastospore and submerged conidia libraries uniquely shared 130 transcripts corresponding to $5 \cdot 4 \%$ of the total unigene set. A cell wall $\beta$-glucan synthase component was shared, as was a transcript with similarity to the fruit fly, Drosophila melanogaster, salivary glue protein Sgs3. The latter protein is a member of a family of glycosylated proteins that are part of the salivary glue secreted by larvae as a means of fixing themselves to an external substrate, and this transcript may encode for a novel type of fungal surface adhesion protein. Both cell types expressed two different free radical detoxifying enzymes, a Mn-superoxide dismutase and $\mathrm{Cu}-\mathrm{Zn}$-superoxide dismutase, a phenol detoxifying enzyme (phenol 2-monooxygenase) and an EST with similarity to enzymes (gibberellin 2 - $\beta$-dioxygenases) that modify or inactivate the gibberellin family of plant signalling compounds. Blastospore and submerged conidia shared transcripts also included a vegetative incompatibility gene and a moderately to highly represented EST (transcript found 5 and 8 times in the blastospore and submerged conidia libraries, respectively) similar to a dehydroquinate synthase family enzyme (2-epi-5-valiolone synthase), possibly involved in the biosynthesis of amylostatin/aminoglycoside-like antibiotics.

Aerial conidia and submerged conidia shared 146 transcripts (6\% of the total), although a number of these transcripts corresponded to housekeeping genes such as ribosomal components, transcription factors and metabolic enzymes. Common transcripts between the aerial conidia and submerged conidia included a putative cuticle-degrading (serine) protease, and a number of ESTs responsible for nutrient uptake, including an amino acid permease similar to a transporter induced by the fungal biocontrol agent Trichoderma harzianum during mycoparasitism, a highaffinity glucose transporter and a galactose-proton symporter. In addition, an EST similar to $14-3-3$ proteins, a family of conserved small acidic proteins that have been implicated in playing major roles in a wide variety of signalling cascades and that are essential for normal hyphal and cell development, was identified.

ESTs found in all three libraries accounted for $6.5 \%$ of the total unique set. Many of these transcripts coded for ribosomal proteins, histones, protein-turnover enzymes, and basic metabolic and ATP generating enzymes. As noted before, one of the most abundant transcripts found in all three libraries was a hydrophobin similar to the Aspergillus rodlet-layer protein. Hydrophobins have been best characterized as being the major protein constituent of the rodlet layer (outer cell envelope layer) found on aerial conidia; however, they have also been implicated in hyphal development and virulence (Kazmierczak et al., 2005; Linder et al., 2005). Hydrophobins can also be secreted into the media where they self-assemble into $2 \mathrm{D}$ arrays at water-air interfaces that act to decrease the surface tension of water, enabling hyphae and other structures to grow into the air (Talbot, 1999; Wosten et al., 1999).

An EST similar to the Malassezia furfur major allergen Malf1 (Schmidt et al., 1997) was also found in all three libraries and may correspond to one of the $B$. bassiana antigens previously identified in Western blots of fungal extracts probed with human sera (Westwood et al., 2005). A covalently linked cell wall protein belonging to the PIR (proteins with internal repeats) family was moderately represented in the submerged conidia library (six times), and also found in the aerial conidia and blastospore libraries (twice and once, respectively). A number of depolymerases likely to function in general processes were also represented in all three libraries. These included a vacuolar aspartate protease similar to saccharopepsin or protease A, implicated in the posttranslational regulation of S. cerevisiae vacuolar proteinases, a transcript similar to the S. cerevisiae cytoplasm to vacuole targeting lipase, and an EST similar to a $S$. cerevisiae cell wall glycoprotein that displays lectin-like binding to $1,3-\beta$-glucan and chitin, and possesses $1,3-\beta$ exoglucanase activity with laminarin as substrate (Klebl \& Tanner, 1989; Teter et al., 2001; Woolford et al., 1986).

\section{Conclusions}

A small number of transcriptome analyses have been reported for filamentous fungi, particularly with respect to plant and arthropod pathogens. Transcript analyses performed on distinct developmental stages (germinating teliospores, diploid filamentous growth and microsclerotia development) or during pathogenic growth of the plant pathogens Ustilago maydis and Verticillium dahliae have provided bases for genome annotation and further exploration of these fungi (Neumann \& Dobinson, 2003; Nugent et al., 2004; Sacadura \& Saville, 2003). EST analyses of two subspecies of the entomopathogenic fungus Metarhizium anisopliae revealed distinct patterns of expression of secreted proteases and pathogenicity factors that have led to the ability to examine gene expression patterns during infection of various insect hosts (Freimoser et al., 2003 , 2005). Our analyses revealed a robust and dynamic gene expression repertoire available to the entomopathogenic fungus $B$. bassiana. Aerial conidia, in vitro blastospores and submerged conidia are morphologically and biochemically different, a distinction borne out through the analysis of their transcripts. The EST collection offers an insight into components that may aid the fungus in the process of insect virulence. A large number of depolymerases including 
proteases, glycosidases and lipases were identified, as were numerous carbohydrate and amino acid transporters. Similarity based searches identified transcripts corresponding to the production of toxic secondary metabolites, including a putative sterigmatocystin gene cluster. The EST collection also contained a large variety of expressed genes encoding free radical scavenging, antioxidant proteins, including superoxide dismutases, catalases and peroxidases. These proteins have been implicated in providing protection against host oxidative defence systems and are important components mediating fungal virulence. Furthermore, a large number of ESTs similar to efflux or multidrug transport systems as well as numerous cytochrome p450s similar to xenobiotic and antimicrobial-detoxifying enzymes were noted, and may help account for the remarkable antibiotic resistance of Beauveria species. B. bassiana ESTs were also identified encoding a number of potential antimicrobial compounds, including lysozyme and an antimicrobial peptide that may defend against endogenous microflora or secondary colonizers. It should be noted that the room for novel gene discovery remains quite vast since the annotated portion of the EST collection represents only $\sim 50 \%$ of the total. From approximately 7500 clones, 2400 unique sequences were identified, although the absolute number might be lower (10-15\%) due to failure of non-overlapping sequences and splice variants to cluster, but still represents a significant portion of the genome. Further sequencing of the libraries should yield additional new transcripts.

\section{ACKNOWLEDGEMENTS}

We are indebted to O'Neil Shand, Ines Robliano and Brett Kirkland for help in the data analysis. This work was supported in part by funds made available from the University of Florida, IFAS and NSF reseach grant \# EF-0412137.

\section{REFERENCES}

Adachi, H., Tsujimoto, M., Fukasawa, M., Sato, Y., Arai, H., Inoue, K. \& Nishimura, T. (1997). cDNA cloning and expression of chicken aminopeptidase $\mathrm{H}$, possessing endopeptidase as well as aminopeptidase activity. Eur J Biochem 245, 283-288.

Anderson, S. O., Hojrup, P. \& Roepstorff, P. (1995). Insect cuticular proteins. Insect Biochem Mol Biol 25, 153-176.

August, P. R., Flickinger, M. C. \& Sherman, D. H. (1994). Cloning and analysis of a locus $(\mathrm{mcr})$ involved in mitomycin $\mathrm{C}$ resistance in Streptomyces lavendulae. J Bacteriol 176, 4448-4454.

Barbault, F., Landon, C., Guenneugues, M., Meyer, J. P., Schott, V., Dimarcq, J. L. \& Vovelle, F. (2003). Solution structure of Alo-3: a new knottin-type antifungal peptide from the insect Acrocinus longimanus. Biochemistry 42, 14434-14442.

Basrai, M. A., Lubkowitz, M. A., Perry, J. R., Miller, D., Krainer, E., Naider, F. \& Becker, J. M. (1995). Cloning of a Candida albicans peptide transport gene. Microbiology 141, 1147-1156.

Bidochka, M. J., Pfeifer, T. A. \& Khachatourians, G. G. (1987). Development of the entomopathogenic fungus Beauveria bassiana in liquid cultures. Mycopathologia 99, 77-83.
Bing, L. A. \& Lewis, L. C. (1992). Endophytic Beauveria bassiana (Balsamo) vuillemin in corn: the influence of the plant growth stage and Ostrinia nubilalis (Huebner). Biocontrol Sci Technol 2, 39-47.

Binnington, K. \& Retnakaran, A. (1991). Physiology of the Insect Epidermis. Melbourne: CSIRO Publications.

Blanford, S., Chan, B. H., Jenkins, N., Sim, D., Turner, R. J., Read, A. F. \& Thomas, M. B. (2005). Fungal pathogen reduces potential for malaria transmission. Science 308, 1638-1641.

Boman, H. G. (1981). Insect responses to microbial infections. In Microbial Control of Pests and Plant Diseases, pp. 769-784. Edited by H. D. Burges. New York: Academic Press.

Boucias, D. \& Pendland, J. (1991). Attachment of mycopathogens to cuticle. In the Fungal Spore and Disease Initiation in Plants and Animals, pp. 101-127. Edited by G. T. Cole \& H. C. Hoch. New York: Plenum.

Brown, D. W., Yu, J.-H., Kelkar, H. S., Fernandes, M., Nesbitt, T. C., Keller, N. P., Adams, T. H. \& Leonard, T. J. (1996). Twenty-five coregulated transcripts define a sterigmatocystin gene cluster in Aspergillus nidulans. Proc Natl Acad Sci U S A 93, 1418-1422.

Carruthers, R. I. \& Soper, R. S. (1987). Fungal diseases. In Epizootiology of Insect Diseases, pp. 357-416. Edited by J. R. Fuxa \& Y. Tanada. New York: Wiley.

Charnley, A. K. (1990). Mechanisms of fungal pathogenesis in insects. In Biotechnology of Fungi for Improving Plant Growth, pp. 85-125. Edited by J. M. Whipps \& R. D. Lumsden. New York: Plenum.

Charnley, A. K. \& St Leger, R. (1991). The role of cuticle degrading enzymes in fungal pathogenesis of insects. In The Fungal Spore and Disease Initiation, pp. 267-286. Edited by G. T. Cole \& H. C. Hoch. New York: Plenum.

Clarkson, J. M. \& Charnley, A. K. (1996). New insights into the mechanisms of fungal pathogenesis in insects. Trends Microbiol 4, 197-203.

Desgranges, C., Vergoignan, C., Lereec, A., Riba, G. \& Durand, A. (1993). Use of solid state fermentation to produce Beauveria bassiana for the biological control of European corn borer. Biotechnol Adv 11, 577-587.

Elliot, S. L., Sabelis, M. W., Janssen, A., van der Geest, L. P. S., Beerling, E. A. M. \& Fransen, J. (2000). Can plants use entomopathogens as bodyguards? Ecol Lett 3, 228-235.

El-Sayed, G. N., Ignoffo, C. M., Leathers, T. D. \& Gupta, S. C. (1993), Effects of cuticle source and concentration on the expression of hydrolytic enzymes by an entomopathogenic fungus, Nomuraea rileyi. Mycopathologia 122, 149-152.

Farmerie, W., Hammer, J., Liu, L., Sahni, A. \& Scneider, M. (2005). Biological workflow with BlastQuest. Data Knowledge Engineer 53, 75-97.

Ferron, P. (1981). Pest control by the fungi Beauveria and Metarhizium. In Microbial Control of Pests and Plant Diseases 1970-1980, pp. 465-482. Edited by H. D. Burges. New York: Academic Press.

Freimoser, F. M., Screen, S., Bagga, S., Hu, G. \& St Leger, R. J. (2003). Expressed sequence tag (EST) analysis of two subspecies of Metarhizium anisopliae reveals a plethora of secreted proteins with potential activity in insect hosts. Microbiology 149, 239-247.

Freimoser, F. M., Hu, G. \& St Leger, R. J. (2005). Variation in gene expression patterns as the insect pathogen Metarhizium anisopliae adapts to different host cuticles or nutrient deprivation in vitro. Microbiology 151, 361-371.

Goettel, M. S., Inglis, G. D. \& Wraight, S. P. (2000). Fungi. In Field Manual of Techniques in Invertebrate Pathology, pp. 255-282. Edited by L. A. Lacey \& H. K. Kaya. Dordrecht: Kluwer. 
Gupta, S. C., Leathers, T. D., El-Sayed, G. N. \& Ignoffo, C. M. (1994). Relationships among enzyme activities and virulence parameters in Beaveria bassiana infections of Galleria mellonella and Trichoplusia ni. J Invertebr Pathol 64, 13-17.

Hackman, R. H. (1984). Cuticle: biochemistry. In Biology of the Integument, pp. 583-610. Edited by J. Bereiter-Hahn, A. G. Mateltsy \& K. S. Richards. Berlin: Springer.

Harris, M. A., Clark, J., Ireland, A. \& 56 other authors (2004). The Gene Ontology (GO) database and informatics resource. Nucleic Acids Res 32, D258-D261.

Hegedus, D. D., Bidochka, M. J., Miranpuri, G. S. \& Khachatourians, G. G. (1992). A comparison of the virulence, stability, and cell-wallsurface characteristics of three spore types produced by the entomopathogenic fungus Beauveria bassiana. Appl Microbiol Biotechnol 36, 785-789.

Hirata, D., Fukui, S. \& Yamashita, I. (1988). Nucleotide sequence of the secretable acid protease gene PEPI in the yeast Saccharomycopsis fibuligera. Agric Biol Chem 52, 2647-2649.

Holder, D. J. \& Keyhani, N. O. (2005). Adhesion of the entomopathogenic fungus Beauveria (Cordyceps) bassiana to substrata. Appl Environ Microbiol 71, 5260-5266.

Homann, V., Mende, K., Arntz, C., Ilardi, V., Macino, G., Morelli, G., Bose, G. \& Tudzynski, B. (1996). The isoprenoid pathway: cloning and characterization of fungal FPPS genes. Curr Genet 30, 232-239.

Inglis, G. D., Goettel, M. S. \& Johnson, D. L. (1995). Influence of ultraviolet light protectants on persistence of the entomopathogenic fungus Beauveria bassiana. Biol Control 5, 581-590.

Jeffs, L. B., Xavier, I. J., Matai, R. E. \& Khachatourians, G. G. (1999). Relationships between fungal spore morphologies and surface properties for entomopathogenic members of the genera Beauveria, Metarhizium, Paecilomyces, Tolypocladium, and Verticillium. Can J Microbiol 45, 936-948.

Kazmierczak, P., Kim, D. H., Turina, M. \& Van Alfen, N. K. (2005). A hydrophobin of the chestnut blight fungus, Cryphonectria parasitica, is required for stromal pustule eruption. Eukaryot Cell 4, 931-936.

Khachatourians, G. G. (1996). Biochemistry and molecular biology of entomopathogenic fungi. In The Mycota VI: Human and Animal Relationships, pp. 331-363. Edited by D. H. Howard \& J. D. Miller. Berlin \& Heidelberg: Springer.

Kirkland, B. H., Westwood, G. S. \& Keyhani, N. O. (2004). Pathogenicity of entomopathogenic fungi Beauveria bassiana and Metarhizium anisopliae to ixodidae tick species Dermacentor variabilis, Rhipicephalus sanguineus, and Ixodes scapularis. J Med Entomol 41, 705-711.

Klebl, F. \& Tanner, W. (1989). Molecular cloning of a cell wall exo$\beta$-1,3-glucanase from Saccharomyces cerevisiae. J Bacteriol 171, 6259-6264.

Knight, L. P., Primiano, T., Groopman, J. D., Kensler, T. W. \& Sutter, T. R. (1999). cDNA cloning, expression and activity of a second human aflatoxin $B_{1}$-metabolizing member of the aldo-keto reductase superfamily, AKR7A3. Carcinogenesis 20, 1215-1223.

Lewis, L. C., Bruck, D. J., Gunnarson, R. D. \& Bidne, K. G. (2001). Assessment of plant pathogenicity of endophytic Beauveria bassiana in Bt transgenic and non-transgenic corn. Crop Sci 41, 1395-1400.

Linder, M. B., Szilvay, G. R., Nakari-Setala, T. \& Penttila, M. E. (2005). Hydrophobins: the protein-amphiphiles of filamentous fungi. FEMS Microbiol Rev 29, 877-896.

Lord, J. C. (2001). Desiccant dusts synergize the effect of Beauveria bassiana (Hyphomycetes: moniliales) on stored-grain beetles. J Econ Entomol 94, 367-372.

Maclver, B., McHale, R. H., Saul, D. J. \& Bergquist, P. L. (1994). Cloning and sequencing of a serine proteinase gene from a thermophilic Bacillus species and its expression in Escherichia coli. Appl Environ Microbiol 60, 3981-3988.

MacLeod, D. M. (1954). Investigations on the genera Beauveria Vuil and Tritirachium Limber. Can J Bot 32, 818-890.

Martin, V., Ribas, J. C., Carnero, E., Duran, A. \& Sanchez, Y. (2000). $b g s 2^{+}$, a sporulation-specific glucan synthase homologue is required for proper ascospore wall maturation in fission yeast. Mol Microbiol 38, 308-321.

Martinez, J. L., Herrero, M. \& de Lorenzo, V. (1994). The organization of intercistronic regions of the aerobactin operon of pColV-K30 may account for the differential expression of the iucABCD iutA genes. J Mol Biol 238, 288-293.

Masuda, N., Kitamura, N. \& Saito, K. (1991). Primary structure of protein moiety of Penicillium notatum phospholipase B deduced from the cDNA. Eur J Biochem 202, 783-787.

Nagao, K., Taguchi, Y., Arioka, M., Kadokura, H., Takatsuki, A., Yoda, K. \& Yamasaki, M. (1995). $b f r 1^{+}$, a novel gene of Schizosaccharomyces pombe which confers brefeldin A resistance, is structurally related to the ATP-binding cassette superfamily. $J$ Bacteriol 177, 1536-1543.

Neumann, M. J. \& Dobinson, K. F. (2003). Sequence tag analysis of gene expression during pathogenic growth and microsclerotia development in the vascular wilt pathogen Verticillium dahliae. Fungal Genet Biol 38, 54-62.

Neville, A. C. (1975). Biology of the Arthropod Cuticle. Berlin: Springer.

Nugent, K. G., Choffe, K. \& Saville, B. J. (2004). Gene expression during Ustilago maydis diploid filamentous growth: EST library creation and analyses. Fungal Genet Biol 41, 349-360.

Pendland, J. C. \& Boucias, D. G. (1985). Hemagglutinin activity in the hemolymph of Anticarsia gemmatalis larvae infected with the fungus Nomuraea rileyi. Dev Comp Immunol 9, 21-30.

Pendland, J. C. \& Boucias, D. G. (1986). Characteristics of a galactose-binding hemagglutinin (lectin) from hemolymph of Spodoptera exigua larvae. Dev Comp Immunol 10, 477-487.

Pendland, J. C., Hung, S. Y. \& Boucias, D. G. (1993). Evasion of host defense by in vivo-produced protoplast-like cells of the insect mycopathogen Beauveria bassiana. J Bacteriol 175, 5962-5969.

Peraza, L. \& Hansberg, W. (2002). Neurospora crassa catalases, singlet oxygen and cell differentiation. Biol Chem 383, 569-575.

Pfeifer, T. A. \& Khachatourians, G. G. (1993). Electrophoretic karyotype of the entomopathogenic deuteromycete Beauveria bassiana. J Invertebr Pathol 61, 231-235.

Renobales, M., Nelson, D. R. \& Blomquist, G. J. (1991). Cuticular lipids. In Physiology of the Insect Epidermis, pp. 240-251. Edited by K. Binnington \& A. Retnakaran. Melbourne: CSIRO Publications.

Riley, P. A. (1997). Melanin. Int J Biochem Cell Biol 29, 1235-1239.

Sacadura, N. T. \& Saville, B. J. (2003). Gene expression and EST analyses of Ustilago maydis germinating teliospores. Fungal Genet Biol 40, 47-64.

Saupe, S., Turcq, B. \& Begueret, J. (1995). A gene responsible for vegetative incompatibility in the fungus Podospora anserina encodes a protein with a GTP-binding motif and G $\beta$ homologous domain. Gene 162, 135-139.

Schmidt, M., Zargari, A., Holt, P., Lindbom, L., Hellman, U., Whitley, P., van der Ploeg, I., Härfast, B. \& Scheynius, A. (1997). The complete cDNA sequence and expression of the first major allergenic protein of Malassezia furfur, Mal f 1 . Eur J Biochem 246, 181-185.

Scholte, E. J., Ng'habi, K., Kihonda, J., Takken, W., Paaijmans, K., Abdulla, S., Killeen, G. F. \& Knols, B. G. (2005). An entomopathogenic 
fungus for control of adult African malaria mosquitoes. Science 308, 1641-1642.

St Leger, R. (1991). Integument as a barrier to microbial infections. In Physiology of the Insect Epidermis, pp. 284-306. Edited by $\mathrm{K}$. Binnington \& A. Retnakaran. Melbourne: CSIRO Publications.

Talbot, N. J. (1999). Fungal biology. Coming up for air and sporulation. Nature 398, 295-296.

Talibi, D. \& Raymond, M. (1999). Isolation of a putative Candida albicans transcriptional regulator involved in pleiotropic drug resistance by functional complementation of a $p d r 1 p d r 3$ mutation in Saccharomyces cerevisiae. J Bacteriol 181, 231-240.

Tercero, J. A., Lacalle, R. A. \& Jimenez, A. (1993). The pur8 gene from the pur cluster of Streptomyces alboniger encodes a highly hydrophobic polypeptide which confers resistance to puromycin. Eur J Biochem 218, 963-971.

Teter, S. A., Eggerton, K. P., Scott, S. V., Kim, J., Fischer, A. M. \& Klionsky, D. J. (2001). Degradation of lipid vesicles in the yeast vacuole requires function of Cvt17, a putative lipase. J Biol Chem 276, 2083-2087.

Thomas, K. C., Khachatourians, G. G. \& Ingledew, W. M. (1987). Production and properties of Beauveria bassiana conidia cultivated in submerged culture. Can J Microbiol 33, 12-20.

Tjoelker, L. W., Eberhardt, C., Unger, J., Trong, H. L., Zimmerman, G. A., McIntyre, T. M., Stafforini, D. M., Prescott, S. M. \& Gray, P. W. (1995). Plasma platelet-activating factor acetylhydrolase is a secreted phospholipase A2 with a catalytic triad. J Biol Chem 270, 25481-25487.

Viaud, M., Couteaudier, Y., Levis, C. \& Riba, G. (1996). Genome organization in Beauveria bassiana: electrophoretic karyotype, gene mapping and telomeric fingerprints. Fungal Genet Biol 20, 175-183.
Wagner, B. L. \& Lewis, L. C. (2000). Colonization of corn, Zea mays, by the entomopathogenic fungus Beauveria bassiana. Appl Environ Microbiol 66, 3468-3473.

Westwood, G. S., Huang, S. W. \& Keyhani, N. O. (2005). Allergens of the entomopathogenic fungus Beauveria bassiana. Clin Mol Allergy 3, 1.

White, J. F., Belanger, F., Meyer, W., Sullivan, R. F., Bischoff, J. F. \& Lewis, E. A. (2002). Clavicipitalean fungal epibionts and endophytes - development of symbiotic interactions with plants. Symbiosis 33, 201-213.

Woolford, C. A., Daniels, L. B., Park, F. J., Jones, E. W., Van Arsdell, J. N. \& Innis, M. A. (1986). The PEP4 gene encodes an aspartyl protease implicated in the posttranslational regulation of Saccharomyces cerevisiae vacuolar hydrolases. Mol Cell Biol 6, 2500-2510.

Wosten, H. A. (2001). Hydrophobins: multipurpose proteins. Annu Rev Microbiol 55, 625-646.

Wosten, H. A., van Wetter, M. A., Lugones, L. G., van der Mei, H. C., Busscher, H. J. \& Wessels, J. G. (1999). How a fungus escapes the water to grow into the air. Curr Biol 9, 85-88.

Wraight, S. P., Jackson, M. A. \& de Kock, S. L. (2001). Production, stabilization, and formulation of fungal biocontrol agents. In Fungi as Biocontrol Agents: Progress, Problems and Potential, pp. 253-287. Edited by T. M. Butt, C. Jackson \& N. Magan. Wallingford: CAB International.

Yanai, K., Kojima, N., Takaya, N., Horiuchi, H., Ohta, A. \& Takagi, M. (1994). Isolation and characterization of two chitin synthase genes from Aspergillus nidulans. Biosci Biotechnol Biochem 58, 1828-1835.

You, I. S., Ghosal, D. \& Gunsalus, I. C. (1991). Nucleotide sequence analysis of the Pseudomonas putida PpG7 salicylate hydroxylase gene (nahG) and its 3'-flanking region. Biochemistry 30, 1635-1641. 